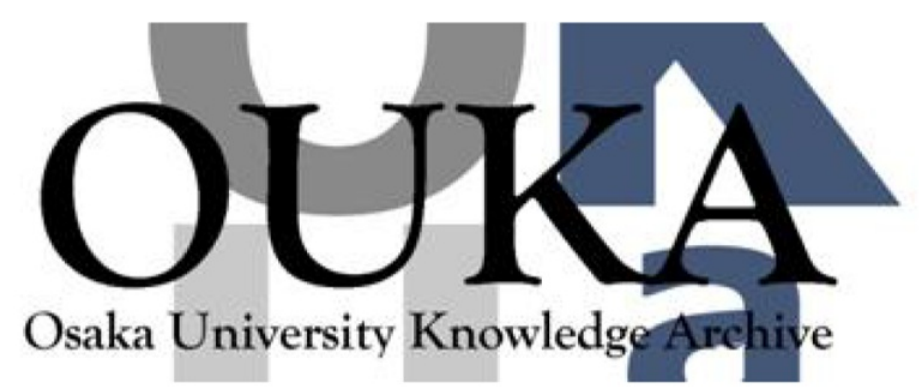

\begin{tabular}{|c|l|}
\hline Title & $\begin{array}{l}\text { Complex formation of a triple-helical peptide } \\
\text { with sodium heparin }\end{array}$ \\
\hline Author(s) & Ishida, Saki; Yoshida, Tasuku; Terao, Ken \\
\hline Citation & Polymer Journal. 51(11) p. 1181-p. 1187 \\
\hline Issue Date & $2019-11$ \\
\hline oaire:version & AM \\
\hline URL & https://hdl. handle. net/11094/77641 \\
\hline rights & \\
\hline Note & \\
\hline
\end{tabular}

Osaka University Knowledge Archive : OUKA

https://ir. Library. osaka-u. ac. jp/

Osaka University 


\title{
Complex formation of a triple-helical peptide with sodium heparin
}

\author{
Saki Ishida, ${ }^{1}$ Tasuku Yoshida, ${ }^{1}$ and Ken Terao ${ }^{1}$ \\ ${ }^{1}$ Department of Macromolecular Science, Graduate School of Science, Osaka University, 1-1 \\ Machikaneyama-cho, Toyonaka, Osaka 560-0043, Japan. \\ E-mail: kterao@chem.sci.osaka-u.ac.jp (Ken Terao) \\ Running Head: Complex formation of collagen peptide and heparin
}

\begin{abstract}
Small-angle X-ray scattering (SAXS) and circular dichroism measurements were carried out for NaHeparin and a triple helical peptide, H-(Pro-Pro-Gly) $10-\mathrm{OH}$ (PPG10), in aqueous sodium chloride $(\mathrm{NaCl})$ at ionic strengths of $20 \mathrm{mM}, 50 \mathrm{mM}$, and $150 \mathrm{mM}$ at different temperatures. While PPG10 forms a triple helix below $25^{\circ} \mathrm{C}$, the melting temperature of the triple helix in the mixed solution is considerably higher $\left(\sim 10{ }^{\circ} \mathrm{C}\right)$ at low $C_{\mathrm{S}}$ values than without NaHeparin. Part of the PPG10 molecules formed complexes with NaHeparin in $20 \mathrm{mM}$ and $50 \mathrm{mM}$ aqueous $\mathrm{NaCl}$ at 15 ${ }^{\circ} \mathrm{C}$, but all solutes were molecularly dispersed at $75{ }^{\circ} \mathrm{C}$, indicating that only triple helices form complexes with NaHeparin. Electrostatic attraction plays an important role in the complexation, since no complex formation was observed in $150 \mathrm{mM}$ aqueous $\mathrm{NaCl}$. The scattering function of the complex was explained by the presence of a thick wormlike chain, indicating that the molecular shape is different from that of the previously investigated complex with polyacrylic acid and carboxymethyl amylose. This suggests appreciable attractive interaction between the triple helical part of PPG10 and NaHeparin.
\end{abstract}

Keywords: anionic polysaccharide / circular dichroism / collagen / electrostatic interactions / small-angle X-ray scattering / triple helical peptide

\section{Introduction}

Oligopeptides with a Pro-Pro-Gly sequence exhibit thermoreversible conformational changes in aqueous solution [1,2], and furthermore, trimerization has been observed at low temperatures [3], while different conformations were found after the renaturation of native multiple helical biopolymers, including collagen, schizophyllan [4], and xanthan [5, 6]. Such synthetic peptides have therefore been widely investigated to clarify the structure-function relationship of collagen [7-11]. Thus, these peptides are called collagen model peptides (CMPs) or triple helical peptides. They have been widely investigated for biomedical applications because some CMPs have relatively high stability in mammalian serum and/or plasma [12-17]. CMPs form a uniform triple helix, which was confirmed by single crystal formation [18]; moreover, a rodlike nature of the triple helix was observed in solution at low temperatures by small-angle X-ray scattering (SAXS) [19] and dielectric dispersion [20].

The positively charged N-termini of the triple helices interact much more significantly with anionic charges than do those of the single coil because the N-termini of the triple helices of CMPs are located near each other. Indeed, the triple helical structure is extremely stabilized in the presence of a small amount of polyelectrolytes [21,22]. The obtained complex has a comb-like 
structure, in which the main chain and side chains consist of the polyelectrolyte and triple helical CMPs, respectively. The most important attractive force in the formation of the complex is the electrostatic force because the stability of the complex increases with both an increasing charge density of the polymer chains and a decreasing ionic strength $\left(C_{\mathrm{S}}\right)$ of the aqueous solvent [23]. Although previous studies on synthetic polyelectrolytes have been performed, mainly focusing on sodium acrylate (NaPAA) and carboxymethyl amylose (NaCMA), the investigation of the interaction of CMPs with natural electrolyte polysaccharides is inviting because some biopolymers have specific interactions with other molecules to facilitate their specific functions in vivo.

In this study, we investigated the complex formation as well as the stabilization of the triple helical structure of H-(Pro-Pro-Gly) ${ }_{10}-\mathrm{OH}$ (PPG10) with and without heparin by means of SAXS and circular dichroism (CD) measurements because the former method is appropriate for identifying the conformational properties of nonlinear polymers and polymeric complexes in solution $[24,25]$. The resulting data were analyzed using linear and branched chain models to estimate the molecular shape of the complex. Since heparin is an abundant anionic polysaccharide in mammals and is widely used as a blood thinner, its interaction with CMPs may become a key factor in developing their biomedical applications.

\section{Experimental Procedure Samples}

Commercially available heparin sodium salt (NaHeparin, TCI) and PPG10 (Peptide Institute) were used for this study without further purification. The number of sulfuric acid groups of each disaccharide unit was estimated from ultimate analysis to be 1.57. The number of anionic groups was thus 2.57 for each disaccharide unit, upon taking carboxylic groups into account. The average molar mass $M_{0}$ of the monosaccharide unit was therefore calculated to be $0.260 \mathrm{~kg} \mathrm{~mol}^{-1}$ for NaHeparin. It should be noted that slight differences in the chemical structure do not cause significant errors in the following analysis of the scattering data.

Size-exclusion chromatography (SEC) equipped with multiangle light scattering (MALS) and refractive index $(\mathrm{RI})$ detectors was used for the analysis of the NaHeparin sample in $50 \mathrm{mM}$ aqueous $\mathrm{NaCl}$ at room temperature $\left(\sim 25^{\circ} \mathrm{C}\right)$ on a Shodex GPC-101 SEC system with a Shodex OHpak SB-G guard column and two Shodex OHpak SB-806M HQ SEC columns connected in series, a Wyatt DAWN Heleos II MALS detector, and a Shodex RI-71 RI detector to determine the weight-average molar mass $M_{\mathrm{w}}$ and the dispersity index $\oslash$, defined as the ratio of the $M_{\mathrm{w}}$ to the number average molar mass. The flow rate was set to $1 \mathrm{~mL} \mathrm{~min}{ }^{-1}$. The two values for NaHeparin were determined to be $M_{\mathrm{w}}=18.9 \mathrm{~kg} \mathrm{~mol}^{-1}$ and $Ð=1.09$. The excess refractive index increment for NaHeparin was determined with a Schulz-Cantow-type differential refractometer (Shimadzu) and identified as $0.115 \mathrm{~cm}^{3} \mathrm{~g}^{-1}$ in $50 \mathrm{mM}$ aqueous $\mathrm{NaCl}$ at $25{ }^{\circ} \mathrm{C}$ at the wavelength $\lambda_{0}$ $=658 \mathrm{~nm}$ in a vacuum, which are the same conditions as those of the light source of the MALS detector. The molar mass $M$ of PPG10 was calculated to be $2.531 \mathrm{~kg} \mathrm{~mol}^{-1}$ according to the chemical structure.

\section{Small-angle X-ray Scattering (SAXS)}

Synchrotron-radiation SAXS measurements were carried out for NaHeparin and NaHeparin/PPG10 in $20-150 \mathrm{mM}$ aqueous $\mathrm{NaCl}$ at $15^{\circ} \mathrm{C}$ and $75^{\circ} \mathrm{C}$ at the BL40B2 beamline in SPring-8 (Hyogo, Japan). Some preliminary measurements were also carried out at the BL-10C beamline in KEK-PF (Ibaraki, Japan). The camera length and $\lambda_{0}$ were set to be $4.16 \mathrm{~m}$ and 0.10 $\mathrm{nm}$, respectively. The intensity of the scattered light was integrated by a Rigaku R-AXIS VII 
imaging plate for $180 \mathrm{~s}$. The position of the incident light at the detector and the precise camera length were calibrated with silver behenate. Solvent and solutions with 3 or 4 different total mass concentrations $c_{t}$, which is the sum of the concentrations of NaHeparin and PPG10, were filled in a quartz capillary cell with a diameter of $2 \mathrm{~mm} \phi$ just before each measurement. The range of $c_{\mathrm{t}}$ was chosen to be between 1 and $5 \mathrm{mg} \mathrm{mL}^{-1}$. The scattering intensity at each pixel was calibrated with the direct beam intensity at the lower end of the sample to correct both the incident light intensity and the transmittance. A circular average procedure was employed with SAngler software [26] to determine the scattering intensity $I(q)$ as a function of the magnitude $q$ of the scattering vector. The scattering intensity of the solvent determined with the same capillary cell was subtracted from $I(q)$ to estimate the excess scattering intensity $\Delta I(q)$. The ratio $\Delta z^{2} R_{q} / K$, where $R_{q}$ and $K$ denote the Rayleigh ratio at $q$ and the optical constant, respectively, was estimated from the following relationship, with the subscript $\mathrm{r}$ being the reference value evaluated from the NaHeparin solutions [27]:

$$
\frac{\Delta z^{2} R_{q}}{K}=M_{\mathrm{w}, \mathrm{r}} \Delta z_{\mathrm{r}}^{2}\left[\frac{c_{\mathrm{r}}}{\Delta I_{\mathrm{r}}(q)}\right] \underset{\substack{c_{\mathrm{r} \rightarrow 0} \rightarrow 0 \\ q \rightarrow 0}}{ } \Delta I(q)
$$

where $\Delta z$ is related to

$$
\Delta z=z-\bar{v} \rho_{\mathrm{e}, \mathrm{s}}
$$

Here, $z$ is the number of moles of electrons per unit mass of the solute, $\bar{v}$ is the partial specific volume of the solute, and $\rho_{\mathrm{e}, \mathrm{s}}$ is the electron density of the solvent. We estimated the $\bar{v}$ value of the NeHeparin solution from the specific density increment at the fixed chemical potential. For PPG10, literature values were used for this study [23].

\section{Circular Dichroism (CD)}

CD measurements were performed by a JASCO J720WO spectropolarimeter for PPG10 with or without NaHeparin in 20,50, and $150 \mathrm{mM}$ aqueous $\mathrm{NaCl}$. The sample solution in which $c_{\mathrm{t}}$ was in the range of the SAXS measurements was placed into a rectangular cell with a path length of 1 $\mathrm{mm}$. The temperature of the JASCO PTC-423L Peltier cell holder was increased at a rate of 6.0 ${ }^{\circ} \mathrm{C} / \mathrm{h}$. The ellipticity $\theta$ was determined at $\lambda_{0}=225 \mathrm{~nm}$ and decreased sigmoidally with increasing temperature due to the conformational change of the triple helix to a single coil-like chain. The melting temperature $T_{\mathrm{m}}$ was estimated as the temperature at which the half of PPG10 had a triple helical structure.

\section{Results and Discussion \\ Stability of Triple Helices}

Fig. 1 illustrates the $T_{\mathrm{m}}$ of PPG10 including NaHeparin. The horizontal axis is the charge group ratio $\alpha$, defined as the molar ratio of anionic groups of NaHeparin to N-terminal groups of PPG10. $T_{\mathrm{m}}$ rapidly increased with increasing $\alpha$ at $C_{\mathrm{S}}=20 \mathrm{mM}$, while almost no $\alpha$ dependence was observed at $C_{\mathrm{S}}=150 \mathrm{mM}$, indicating that the stabilization of the triple helical structure with increasing NaHeparin composition becomes much more significant with the decrease in $C_{\mathrm{S}}$. Another interesting point is that the asymptotic $T_{\mathrm{m}}$ at $C_{\mathrm{S}}=20 \mathrm{mM}$ was approximately $10{ }^{\circ} \mathrm{C}$ higher 
than that for the pure PPG10 system. Similar high stabilization has only been found for sodium poly(acrylic acid) and polyvinylamine, for which the line charge density was $3.8 \mathrm{~nm}^{-1}$ [22]. The value can be estimated for NaHeparin to be $4.6 \mathrm{~nm}^{-1}$ from the helix rise per residue evaluated below, revealing that the electrostatic attractive interaction between PPG10 and NaHeparin plays an important role in the stabilization of the triple helices.

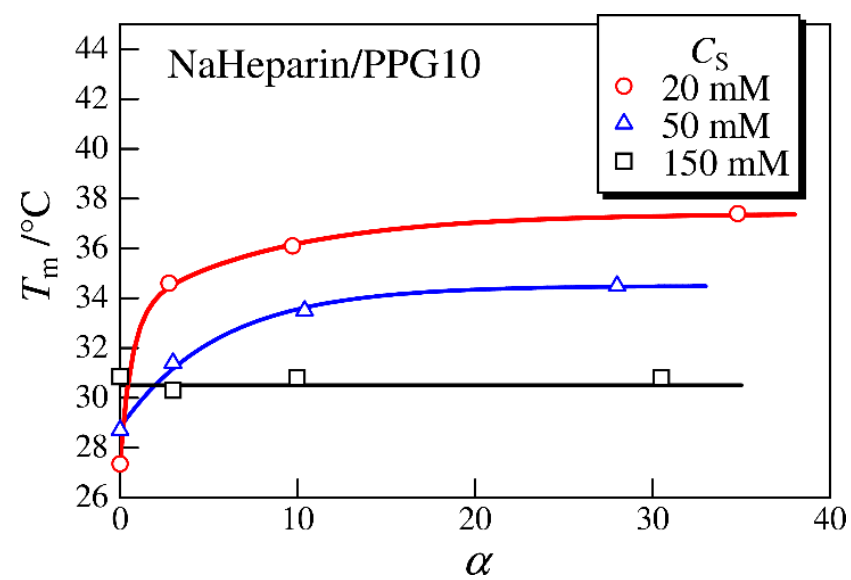

Fig. 1. Composition dependence of $T_{\mathrm{m}}$ for PPG10 including NaHeparin in aqueous $\mathrm{NaCl}$ with $C_{\mathrm{S}}$ $=20 \mathrm{mM}$ (circles), $C_{\mathrm{S}}=50 \mathrm{mM}$ (triangles), and $C_{\mathrm{S}}=150 \mathrm{mM}$ (squares) at a temperature increasing rate of $6{ }^{\circ} \mathrm{C} \mathrm{h}^{-1}$.

\section{Complex Formation of PPG10 and NaHeparin}

The reduced scattering intensity $\Delta z^{2} R_{q} / K c_{\mathrm{t}}$ was almost independent of the $c_{\mathrm{t}}$ at the high $q$ range, suggesting essentially the same complexation within the $c_{\mathrm{t}}$ range investigated $\left(1-5 \mathrm{mg} \mathrm{mL}^{-1}\right)$. The slight decrease in $\Delta z^{2} R_{q} / K c_{\mathrm{t}}$ at the low $q$ range with increasing $c_{\mathrm{t}}$ can be attributed to the second virial term. We thus extrapolated $\Delta z^{2} R_{q} / K c_{\mathrm{t}}$ to $c_{\mathrm{t}}=0$ to eliminate the interparticle interference. The modified square-root Zimm plots for NaHeparin/PPG10 at low $C_{\mathrm{S}}$ and $c_{\mathrm{t}}=0$ are illustrated in Fig. 2, in which the vertical axis is free from $\Delta z$, cf. eq 1 . The scattering intensity at $q=0$ at 15 ${ }^{\circ} \mathrm{C}$ was appreciably lower than that at $75^{\circ} \mathrm{C}$. Considering that $\mathrm{NaHeparin}$ has a much higher molar mass than PPG10, the high scattering intensity at low temperatures indicates complex formation between NaHeparin and PPG10 because this difference is much more significant than that resulting from simply forming the triple helix of PPG10. When we consider that each mixed solution includes three components, PPG10 (CMP), polysaccharides (PS), and their complex, the reduced scattering intensity is expressed as [23]

$$
\left(\frac{M_{\mathrm{PS}}}{n}+\frac{M_{\mathrm{CMP}}}{\alpha}\right)\left(\frac{\Delta z^{2} R_{0}}{K c_{\mathrm{t}}}\right)_{c_{\mathrm{t}}=0}=n\left(\Delta z_{\mathrm{PS}} \frac{M_{\mathrm{PS}}}{n}+\Delta z_{\mathrm{CMP}} M_{\mathrm{CMP}} f\right)^{2}+m \Delta z_{\mathrm{CMP}}^{2} M_{\mathrm{CMP}}{ }^{2}\left(\frac{1}{\alpha}-f\right)
$$

where $n$ and $f$ denote the number of anionic groups of a polysaccharide molecule ( $n=93.5$ for NaHeparin) and the degree of complexation of each polysaccharide chain, with $f \alpha=1$ corresponding to full complexation. The $\left[\left(M_{\mathrm{PS}} / n+M_{\mathrm{CMP}} / \alpha\right)\left(\Delta z^{2} R_{0} / K c_{\mathrm{t}}\right)_{c_{\mathrm{t}}=0}\right]^{1 / 2}$ values are plotted against $1 / \alpha$ in Fig. 3. The plotted data at $C_{\mathrm{S}}=150 \mathrm{mM}$ (panel c) are mostly independent 
of both $1 / \alpha$ and temperature. They are fitted by a straight line calculated by means of eq 3 with $f \alpha$ $=0$ (no complexation), where $m$ was chosen to be 3 and 1 at $15^{\circ} \mathrm{C}$ (dashed blue line) and $75{ }^{\circ} \mathrm{C}$ (solid red lines), respectively. This indicates that NaHeparin does not form an appreciable complex with PPG10 in $150 \mathrm{mM}$ aqueous $\mathrm{NaCl}$. This is consistent with the almost constant $T_{\mathrm{m}}$ in Fig. 1. In contrast, significant temperature-dependent scattering intensity was found at lower $C_{\mathrm{S}}$ values, as illustrated in panels a and $\mathrm{b}$ in Fig. 3. While the data points at $75^{\circ} \mathrm{C}$ are fitted by a dotdashed red lines for $f \alpha=0$ and $m=1$, those at $15^{\circ} \mathrm{C}$ are between $f \alpha=0$ and 1, suggesting partial complexation consisting of NaHeparin and PPG10. If we choose $f \alpha=0.4$, the calculated dashed lines mostly reproduce the experimental data, indicating that some of the peptide molecules form complexes with polysaccharides at $15^{\circ} \mathrm{C}$. It should be noted that it is unclear whether $f \alpha$ is independent of $\alpha$ since sufficient accuracy of $f \alpha$ is only found at the highest $1 / \alpha$. Another important point is that the shape of the molar ellipticity [ $\theta$ ] of PPG10 plotted against the temperature is mostly independent of $\alpha$ and $C_{\mathrm{S}}$, even though a significantly higher $T_{\mathrm{m}}$ was observed in Fig. 1. This is likely because complex formation is dynamic, and the time constant of association is much faster than the temperature change of the $\mathrm{CD}$ measurements.

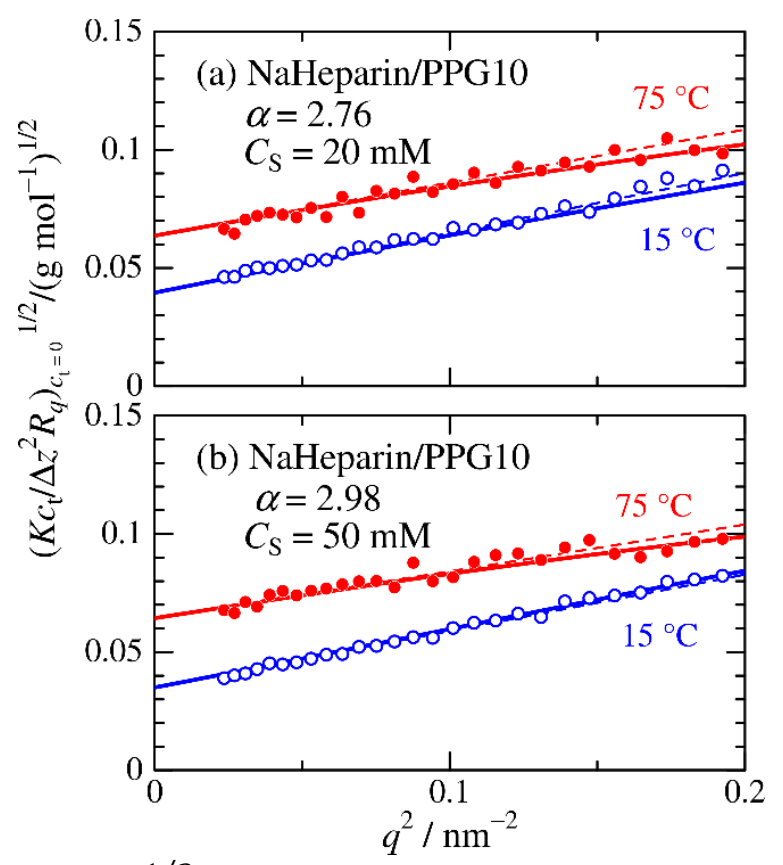

Fig. 2. Plots of $\left(K c_{\mathrm{t}} / \Delta z^{2} R_{q}\right)_{c_{\mathrm{t}}=0}{ }^{1 / 2}$ versus $q^{2}$ in aqueous $\mathrm{NaCl}$ at $15^{\circ} \mathrm{C}$ (unfilled circles) and at $75{ }^{\circ} \mathrm{C}$ (filled circles). The range of $c_{\mathrm{t}}$ was between 1 and $5 \mathrm{mg} \mathrm{mL}^{-1}$. (a) Naheparin/PPG10 ( $\alpha=$ 2.76, $\left.C_{\mathrm{S}}=20 \mathrm{mM}\right)$ and (b) Naheparin/PPG10 $\left(\alpha=2.98, C_{\mathrm{S}}=50 \mathrm{mM}\right)$. 


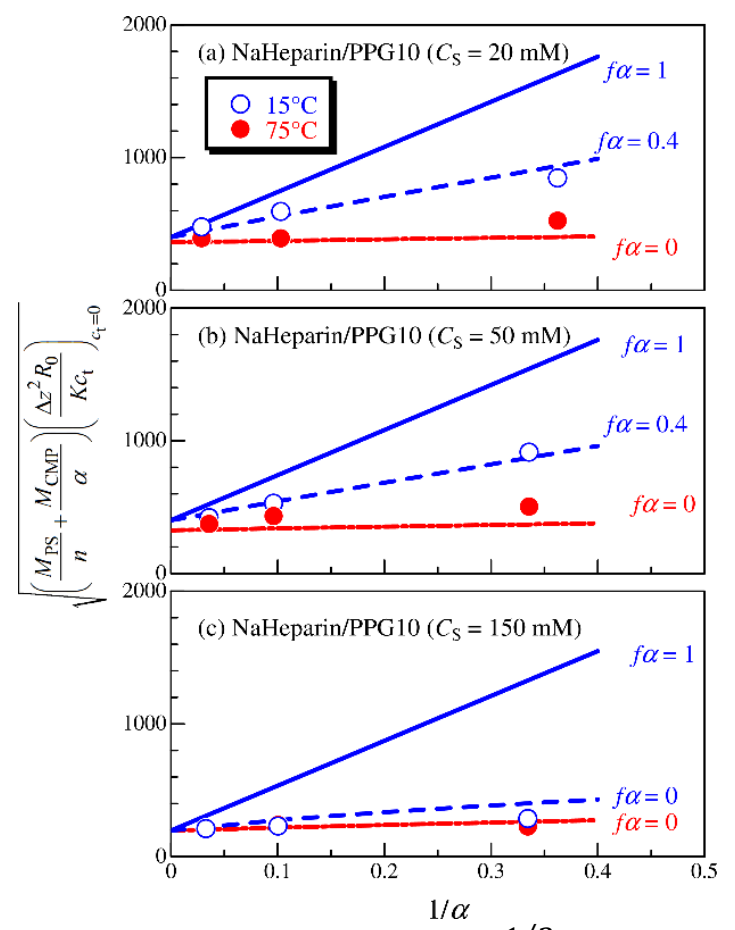

Fig. 3. Plots of $\left[\left(M_{\mathrm{PS}} / n+M_{\mathrm{CMP}} / \alpha\right)\left(\Delta z^{2} R_{0} / K c_{\mathrm{t}}\right)_{c_{\mathrm{t}}=0}\right]^{1 / 2}$ versus $1 / \alpha$ for NaHeparin/PPG10 in aqueous $\mathrm{NaCl}$ at $15{ }^{\circ} \mathrm{C}$ (unfilled circles) and $75{ }^{\circ} \mathrm{C}$ (filled circles). (a) $C_{\mathrm{S}}=20 \mathrm{mM}$, (b) $C_{\mathrm{S}}=50$ $\mathrm{mM}$, and (c) $C_{\mathrm{S}}=150 \mathrm{mM}$. Solid and dashed lines indicate the values calculated for $15{ }^{\circ} \mathrm{C}$ by using eq 3 with the indicated $f \alpha$ and $m=3$ (triple helix). Dot-dashed lines indicate the theoretical values for $f \alpha=0$ and $m=1$ for $75^{\circ} \mathrm{C}$ (single coil).

\section{Molecular Shape of Polysaccharides in Solution}

Prior to discussing the conformation of the complex NaHeparin and PPG10, the conformation of NaHeparin needed to be confirmed. Fig. 4 shows the Holtzer plot obtained from SAXS measurements of NaHeparin in $20 \mathrm{mM}, 50 \mathrm{mM}$, and $150 \mathrm{mM}$ aqueous $\mathrm{NaCl}$. The shape is typical of the wormlike chain with a finite thickness $[28,29]$. It can be seen that the shape does not depend appreciably on the temperature or $C_{\mathrm{S}}$, suggesting a similar conformation in all examined solvent conditions. The data were analyzed in terms of the touched-bead wormlike-chain model for which the particle scattering function $P(q)$ is expressed with the bead diameter $d_{\mathrm{b}}$ as [28-31]

$$
P(q)=9\left(\frac{2}{q d_{\mathrm{b}}}\right)^{6}\left(\sin \frac{q d_{\mathrm{b}}}{2}-\frac{q d_{\mathrm{b}}}{2} \cos \frac{q d_{\mathrm{b}}}{2}\right)^{2} P_{0}(q)
$$

where $P_{0}(q)$ is the particle scattering function of the thin wormlike chain. Numerical values can be calculated from the following equation

$$
P_{0}(q)=\frac{2}{L^{2}} \int_{0}^{L}(L-t) I\left(\lambda^{-1} q ; \lambda t\right) d t
$$


when we apply the approximate expression of the characteristic function $I\left(\lambda^{-1} q ; \lambda t\right)$ of the wormlike chain reported by Nakamura and Norisuye $[32,33]$. Here, $L$ and $\lambda^{-1}$ are the contour length and the Kuhn segment length (or twice the persistence length), respectively. A curve fitting procedure was employed in the data analysis to estimate $L=20 \pm 2 \mathrm{~nm}, \lambda^{-1}=12 \pm 3 \mathrm{~nm}$, and $d_{\mathrm{b}}=$ $1.5 \pm 0.3 \mathrm{~nm}$, while the actual parameters for each system are listed in the figure caption. The chain stiffness $\left(\lambda^{-1}\right)$ is fairly close to the reported value of $\lambda^{-1}=9 \mathrm{~nm}$ in $200 \mathrm{mM}$ aqueous $\mathrm{NaCl}$ [34]. It should be noted that the occurrence of essentially the same $\lambda^{-1}$ value at different $C_{\mathrm{S}}$ values is most likely because the experimental $P(q)$ data is not very different from the rod-limiting value (dashed curve), and hence, the accuracy $( \pm 3 \mathrm{~nm})$ of $\lambda^{-1}$ is not high enough to observe the $C_{\mathrm{S}}$ dependence, even though it has been observed for other polyelectrolytes including sodium hyaluronate [35]. The contour length $h$ (or helix rise) per residue, defined as $h \equiv L M_{0} / M_{\mathrm{w}}$, was calculated to be $0.28 \mathrm{~nm}$. This value is somewhat smaller than $0.4-0.5 \mathrm{~nm}$ for NaHeparin in saline $[34,36]$. A possible reason for this finding is that our analysis is different from that in former studies. In any case, to discuss this difference precisely, data of samples with different $M_{\mathrm{w}}$ will be mandatory.

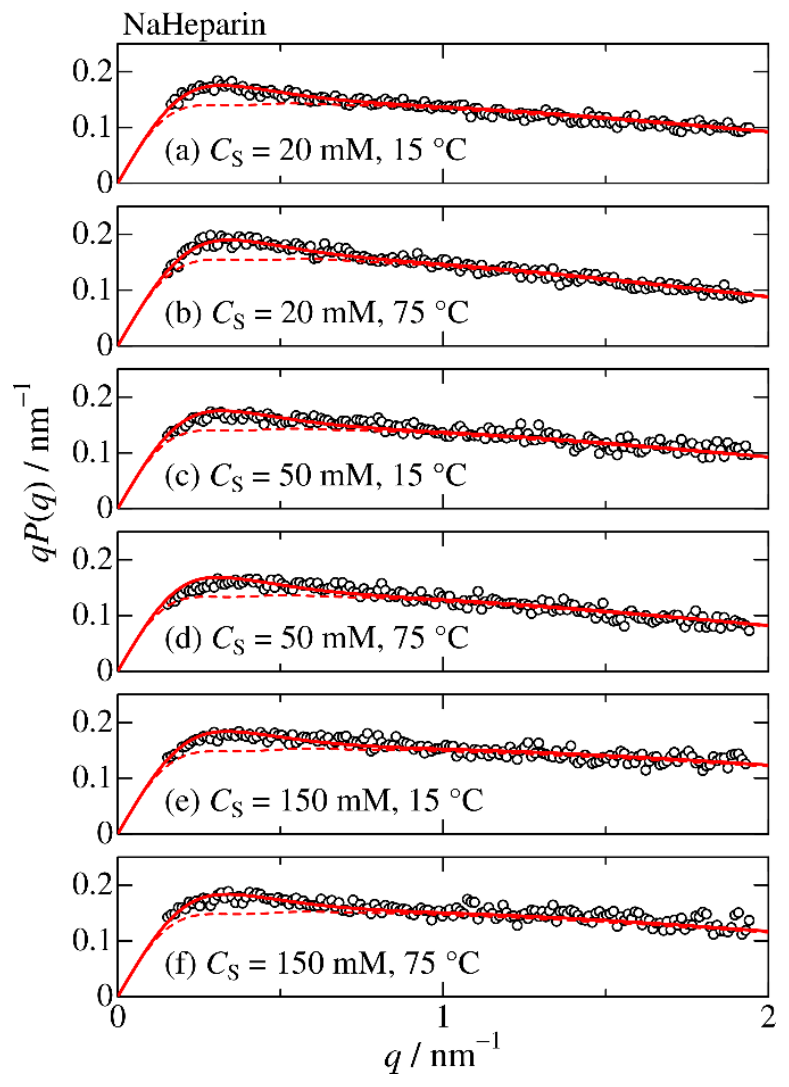

Fig. 4. Holtzer plots of NaHeparin in aqueous NaCl. (a) $C_{\mathrm{S}}=20 \mathrm{mM}$ at $15^{\circ} \mathrm{C}$, (b) $C_{\mathrm{S}}=20 \mathrm{mM}$ at $75^{\circ} \mathrm{C}$, (c) $C_{\mathrm{S}}=50 \mathrm{mM}$ at $15^{\circ} \mathrm{C}$, (d) $C_{\mathrm{S}}=50 \mathrm{mM}$ at $75^{\circ} \mathrm{C}$, (e) $C_{\mathrm{S}}=150 \mathrm{mM}$ at $15^{\circ} \mathrm{C}$, and (f) $C_{\mathrm{S}}$ $=150 \mathrm{mM}$ at $75^{\circ} \mathrm{C}$. Solid curves indicate the theoretical values for the touched-bead wormlike chain with the parameters (a) $L=20 \mathrm{~nm}, \lambda^{-1}=12 \mathrm{~nm}$, and $d_{\mathrm{b}}=1.6 \mathrm{~nm}$, (b) $L=18 \mathrm{~nm}, \lambda^{-1}=12$ $\mathrm{nm}$, and $d_{\mathrm{b}}=1.8 \mathrm{~nm}$, (c) $L=20 \mathrm{~nm}, \lambda^{-1}=12 \mathrm{~nm}$, and $d_{\mathrm{b}}=1.6 \mathrm{~nm}$, (d) $L=21 \mathrm{~nm}, \lambda^{-1}=12 \mathrm{~nm}$, and $d_{\mathrm{b}}=1.7 \mathrm{~nm}$, (e) $L=19 \mathrm{~nm}, \lambda^{-1}=12 \mathrm{~nm}$, and $d_{\mathrm{b}}=1.2 \mathrm{~nm}$, and (f) $L=19 \mathrm{~nm}, \lambda^{-1}=12 \mathrm{~nm}$, and $d_{\mathrm{b}}=1.3 \mathrm{~nm}$. Dashed curves indicate the theoretical values for the rod limit. 


\section{Molecular Shape of the Complex in Solution}

To elucidate the molecular shape of the complex, the Holtzer plots illustrated in Fig. 5 for NeHeparin/PPG10 in $20 \mathrm{mM}$ and $50 \mathrm{mM}$ aqueous $\mathrm{NaCl}$ at $15{ }^{\circ} \mathrm{C}$ were analyzed by the following procedure. The peak position and height are quite similar to those of the single chain in Fig. 4, and the $q P(q)$ values at the higher $q$ range are considerably smaller than those without PPG10. The $P(q)$ data for previously investigated systems - that is, CMP with NaPAA [22] or NaCMA [23] - were well explained in terms of the wormlike comb model, in which the main chain and side chain consist of the polyelectrolyte and CMP, respectively. The current data was therefore compared with the same model. The scattering function $P(q)$ of the mixture of triple helical CMP $\left[P_{\mathrm{CMP}}(q)\right]$ and the wormlike comb [complex, $\left.P_{\text {comp }}(q)\right]$ can be expressed as

$$
P(q)=\frac{n\left(\Delta z_{\mathrm{PS}} \frac{M_{\mathrm{PS}}}{n}+\Delta z_{\mathrm{CMP}} M_{\mathrm{CMP}} f\right)^{2} P_{\text {comp }}(q)+m \Delta z_{\mathrm{CMP}}{ }^{2} M_{\mathrm{CMP}} 2\left(\frac{1}{\alpha}-f\right) P_{\mathrm{CMP}}(q)}{n\left(\Delta z_{\mathrm{PS}} \frac{M_{\mathrm{PS}}}{n}+\Delta z_{\mathrm{CMP}} M_{\mathrm{CMP}} f\right)^{2}+m \Delta z_{\mathrm{CMP}}{ }^{2} M_{\mathrm{CMP}} 2\left(\frac{1}{\alpha}-f\right)}
$$

The scattering function $P_{\mathrm{CMP}}(q)$ of CMP can be expressed as eqs 4 and 5, with $P_{0}(q)$ at an infinitely high $\lambda^{-1}$. $P_{\mathrm{CMP}}(q)$ was thus calculated by the length $L_{\mathrm{CMP}}$ of the triple helical CMP and the diameter of the touched bead $d_{\mathrm{b}}$. The $L_{\mathrm{CMP}}$ value in aqueous media has been determined to be $8.6 \mathrm{~nm}$ via a different method [20]. For the complex, $P_{\text {comp }}(q)$ is a function of the contour length $L_{\mathrm{PS}}$ of the main chain (NeHeparin), the Kuhn segment length $\lambda_{\mathrm{PS}}{ }^{-1}$ of the main chain, $L_{\mathrm{CMP}}, d_{\mathrm{b}}$, and the number of CMP triple helices in each complex, which is $1-5$, depending on $f$ and $\alpha$. Because $d_{\mathrm{b}}$ $=1.5 \mathrm{~nm}$ for NeHeparin is the same as the chain thickness of triple helical CMP [19], we calculated $P(q)$ with eq 6 and $L_{\mathrm{PS}}=20 \mathrm{~nm}, \lambda_{\mathrm{PS}}{ }^{-1}=12 \mathrm{~nm}, L_{\mathrm{CMP}}=8.6 \mathrm{~nm}$, and $d_{\mathrm{b}}=1.5 \mathrm{~nm}$ (no adjustable parameters). The resultant theoretical values shown as blue solid curves in Fig. 4 fairly fit the lowest $q$ region but deviate upward with increasing $q$. If we choose the much larger $d_{\mathrm{b}}$ of $4 \mathrm{~nm}$ for $C_{\mathrm{S}}=20 \mathrm{mM}$ and $5 \mathrm{~nm}$ for $C_{\mathrm{S}}=50 \mathrm{mM}$, the resultant dashed red curves reproduce the experimental data almost quantitatively. This shows that the shape of the complex cannot be explained by the wormlike comb model with universal joints. Possibly, triple helical CMPs are adsorbed parallel to the polysaccharide chain, as schematically illustrated in Fig. 6. If so, the scattering function can be modeled by the thick linear wormlike chain. The local helical conformation of NaHeparin within the complex may be extended by the absorbed CMPs. Thus, when we assume that $P_{\text {comp }}(q)$ is a thick wormlike chain with $L=28 \mathrm{~nm}(h=0.4 \mathrm{~nm}), \lambda^{-1}=12 \mathrm{~nm}$, and $d_{\mathrm{b}}=4-6 \mathrm{~nm}$ depending on the sample, the calculated dot-dashed curves well explain the experimental data. As described above, only about half of the CMPs form complexes with NaHeparin $(f \alpha \sim 0.4)$, whereas the line charge density of NaHeparin is higher than that of the previously investigated NaPAA [22]. This is likely because the absorbed CMPs inhibit further complexation. The similar $f \alpha$ values obtained at different $C_{\mathrm{S}}$ are also due to the inhibition. 


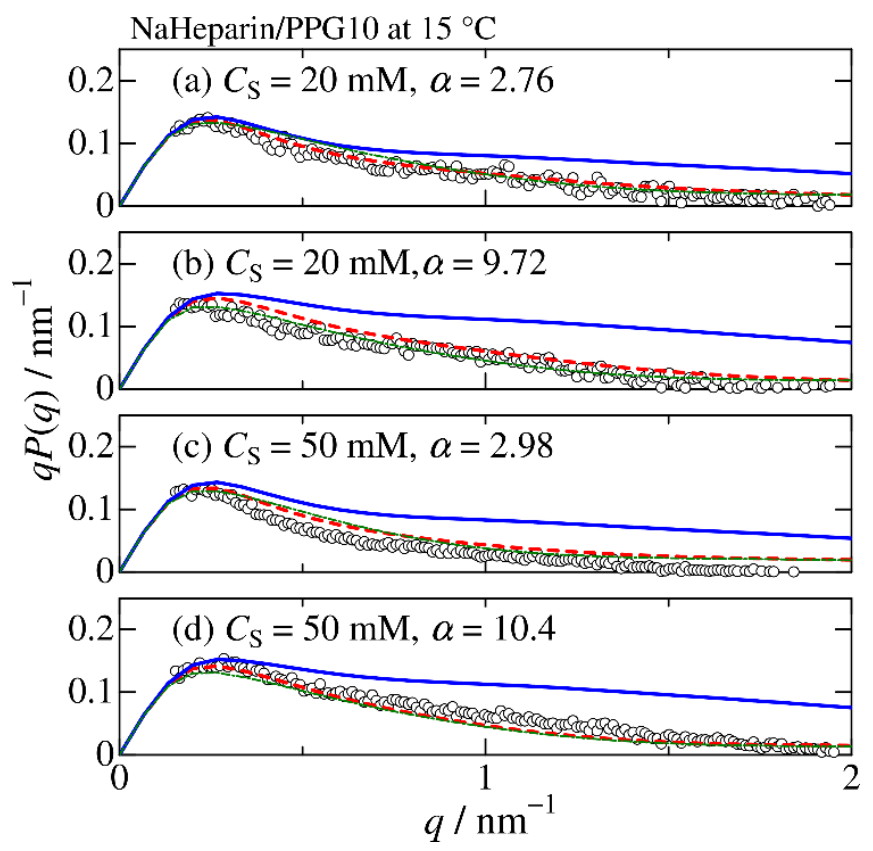

Fig. 5. Holtzer plots of NaHeparin/PPG10 in aqueous $\mathrm{NaCl}$ at $15{ }^{\circ} \mathrm{C}$. (a) $C_{\mathrm{S}}=20 \mathrm{mM}$ and $\alpha=$ 2.76 , (b) $C_{\mathrm{S}}=20 \mathrm{mM}$ and $\alpha=9.72$, (c) $C_{\mathrm{S}}=50 \mathrm{mM}$ and $\alpha=2.98$, (d) $C_{\mathrm{S}}=50 \mathrm{mM}$ and $\alpha=10.4$. Solid curves indicate the theoretical values for touched-bead wormlike comb-like chains (see text). Dashed curves (red) are the theoretical values from the same equation with $d_{\mathrm{b}}=4 \mathrm{~nm}\left(C_{\mathrm{S}}=20\right.$ $\mathrm{mM})$ and $5 \mathrm{~nm}\left(C_{\mathrm{S}}=50 \mathrm{mM}\right)$. Dot-dashed curves (green) are for thick linear wormlike chains (see text).

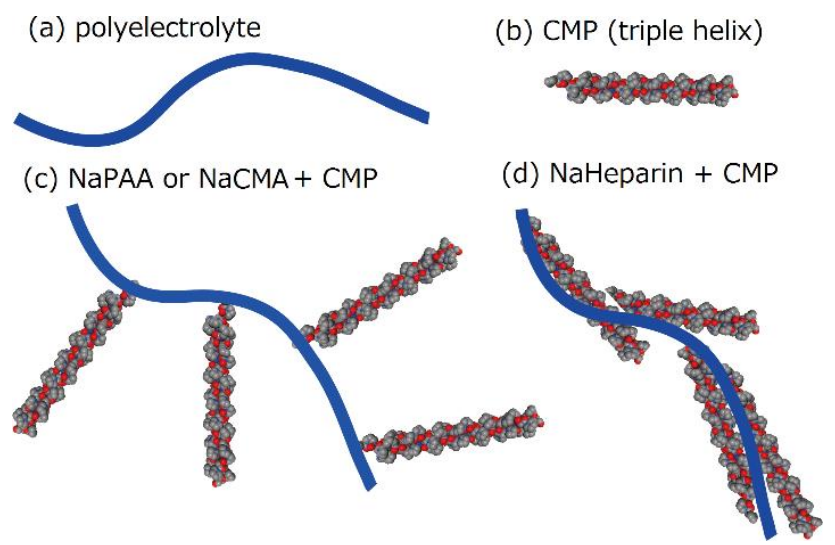

Fig. 6. Schematic representations of possible complexations. (a) Polyelectrolyte chain. (b) Triple helical CMP. (c) Previous studies of polyelectrolytes + CMP. (d) NaHeparin + CMP.

\section{Conclusions}

As in the case of synthetic polyelectrolytes, a natural polysaccharide, NaHeparin, forms a complex with a triple helical collagen model peptide (CMP) at relatively low ionic strengths $(20 \mathrm{mM}$ and $50 \mathrm{mM}$ ) at $15{ }^{\circ} \mathrm{C}$. While the previously investigated sodium polyacrylate and sodium carboxymethyl amylose form comblike complexes with universal joints, the scattering data for NaHeparin/PPG10 may indicate a parallel alignment, suggesting that not only N-termini of CMP but also the triple helical part interact attractively with NaHeparin. This specific interaction between NaHeparin with the current peptide may be a key factor in elucidating the interaction of 
heparin with other extracellular matrices in vivo. Furthermore, it may also be important in clarifying the multilayer formation and functionality of complexes of natural collagen and electrolyte polysaccharides [37-39].

\section{Acknowledgments}

We thank Prof. Takahiro Sato at Osaka University for the fruitful discussion and Dr. Noboru Ohta (SPring-8), Prof. Noriyuki Igarashi (KEK), and Prof. Nobutaka Shimizu (KEK) for the SAXS measurements. The SAXS data were acquired at the BL40B2 beamline in SPring-8 with the approval of the Japan Synchrotron Radiation Research Institute (JASRI) (Proposal Nos. 2015B1100, 2016A1053, and 2016B1088) and at the BL-10C beamline in KEK-PF under the approval of the Photon Factory Program Advisory Committee (Proposal No. 2011G557). This work was partially supported by JSPS KAKENHI Grant Nos. JP17K05884 and JP18H02020.

\section{References}

1. Engel J, Kurtz J, Katchalski E, Berger A. Polymers of tripeptides as collagen models. J Mol Biol. 1966;17:255-72.

2. Sakakibara S, Kishida Y, Kikuchi Y, Sakai R, Kakiuchi K. Synthesis of poly-(L-prolylL-prolylglycyl) of defined molecular weights. Bull Chem Soc Jpn. 1968;41:1273-.

3. Kobayashi Y, Sakai R, Kakiuchi K, Isemura T. Physicochemical analysis of (Pro-ProGly)n with defined molecular weight-temperature dependence of molecular weight in aqueous solution. Biopolymers. 1970;9:415-25.

4. Stokke BT, Elgsaeter A, Brant DA, Kuge T, Kitamura S. Macromolecular cyclization of (1-->6)-branched-(1-->3)-beta-D-glucans observed after denaturation-renaturation of the triple-helical structure. Biopolymers. 1993;33:193-8.

5. Matsuda Y, Sugiura F, Okumura K, Tasaka S. Renaturation behavior of xanthan with high molar mass and wide molar mass distribution. Polym J. 2016;48:653-8.

6. Matsuda Y, Okumura K, Tasaka S. Molar mass dependence of structure of xanthan thermally denatured and renatured in dilute solution. Polym J. 2018;50:1043-9.

7. Shoulders MD, Raines RT. Collagen structure and stability. Annu Rev Biochem. 2009;78:929-58.

8. Berisio R, De Simone A, Ruggiero A, Improta R, Vitagliano L. Role of side chains in collagen triple helix stabilization and partner recognition. J Pept Sci. 2009;15:131-40.

9. Okuyama K. Revisiting the molecular structure of collagen. Connect Tissue Res. 2008;49:299-310.

10. Fields GB. Synthesis and biological applications of collagen-model triple-helical peptides. Organic \& Biomolecular Chemistry. 2010;8:1237-58.

11. Fallas JA, O'Leary LER, Hartgerink JD. Synthetic collagen mimics: self-assembly of homotrimers, heterotrimers and higher order structures. Chem Soc Rev. 2010;39:351027.

12. Fan CY, Huang CC, Chiu WC, Lai CC, Liou GG, Li HC, Chou MY. Production of multivalent protein binders using a self-trimerizing collagen-like peptide scaffold. FASEB J. 2008;22:3795-804.

13. Ndinguri MW, Zheleznyak A, Lauer JL, Anderson CJ, Fields GB. Application of collagen-model triple-helical peptide-amphiphiles for CD44-targeted drug delivery systems. J Drug Deliv. 2012;2012:592602. 
14. Yasui H, Yamazaki CM, Nose H, Awada C, Takao T, Koide T. Potential of collagen-like triple helical peptides as drug carriers: Their in vivo distribution, metabolism, and excretion profiles in rodents. Biopolymers. 2013;100:705-13.

15. Yamazaki CM, Nakase I, Endo H, Kishimoto S, Mashiyama Y, Masuda R, Futaki S, Koide T. Collagen-like cell-penetrating peptides. Angew Chem Int Ed. 2013;52:5497500.

16. Shinde A, Feher KM, Hu C, Slowinska K. Peptide internalization enabled by folding: triple helical cell-penetrating peptides. J Pept Sci. 2015;21:77-84.

17. Bennink LL, Smith DJ, Foss CA, Pomper MG, Li Y, Yu SM. High serum stability of collagen hybridizing peptides and their fluorophore conjugates. Mol Pharm. 2017;14:1906-15.

18. Okuyama K, Okuyama K, Arnott S, Takayanagi M, Kakudo M. Crystal and molecular structure of a collagen-like polypeptide (Pro-Pro-Gly)10. J Mol Biol. 1981;152:427-43.

19. Terao K, Mizuno K, Murashima M, Kita Y, Hongo C, Okuyama K, Norisuye T, Bächinger HP. Chain dimensions and hydration behavior of collagen model peptides in aqueous solution: [Glycyl-4(R)-hydroxyprolyl-4(R)-hydroxyproline](n), [glycylprolyl4(R)-hydroxyproline](n), and some related model peptides. Macromolecules. 2008;41:7203-10.

20. Shikata T, Minakawa A, Okuyama K. Structure, dynamics, and hydration of a collagen model polypeptide, (L-prolyl-L-prolylglycyl)10, in aqueous media: A chemical equilibrium analysis of triple helix-to-single coil transition. J Phys Chem B. 2009;113:14504-12.

21. Kita Y, Terao K, Sato T. Stabilization of the Triple Helical Structure of a Collagen Model Peptide by Complexation with Polyacrylic Acid in Methanol. Kobunshi Ronbunshu. 2010;67:686-9.

22. Terao K, Kanenaga R, Sato T, Mizuno K, Bächinger HP. Complex formation of collagen model peptides with polyelectrolytes and stabilization of the triple helical structure. Macromolecules. 2012;45:392-400.

23. Terao K, Kanenaga R, Yoshida T, Mizuno K, Bächinger HP. Temperature induced complex formation-deformation behavior of collagen model peptides and polyelectrolytes in aqueous solution. Polymer. 2015;64:8-13.

24. Ryoki A, Ida D, Terao K. Scattering function of semi-rigid cyclic polymers analyzed in terms of worm-like rings: cyclic amylose tris(phenylcarbamate) and cyclic amylose tris(n-butylcarbamate). Polym J. 2017;49:633-7.

25. Uramoto K, Takahashi R, Terao K, Sato T. Local and global conformations of flower micelles and flower necklaces formed by an amphiphilic alternating copolymer in aqueous solution. Polym J. 2016;48:863-7.

26. Shimizu N, Yatabe K, Nagatani Y, Saijyo S, Kosuge T, Igarashi N. Software development for analysis of small-angle X-ray scattering data. AIP Conf Proc. 2016;1741:050017.

27. Glatter O, Kratky O. Small Angle X-ray Scattering. London: Academic Press, 1982.

28. Yamakawa H, Yoshizaki T. Helical Wormlike Chains in Polymer Solutions, 2nd ed. Berlin, Germany: Springer, 2016.

29. Nakamura Y, Norisuye T. 2.02 - Polymer Properties in Solutions. In: Editors-in-Chief: Krzysztof M and Martin M, editors. Polymer Science: A Comprehensive Reference. Amsterdam: Elsevier, 2012. pp. 5-32. 
30. Burchard W, Kajiwara K. The statistics of stiff chain molecules. I. The particle scattering factor. Proc R Soc London, Ser A. 1970;316:185-99.

31. Nagasaka K, Yoshizaki T, Shimada J, Yamakawa H. More on the scattering function of helical wormlike chains. Macromolecules. 1991;24:924-31.

32. Nakamura Y, Norisuye T. Brush-like polymers. In: Borsali R and Pecora R, editors. Soft Matter Characterization: Springer Netherlands, 2008. pp. 235-86.

33. Nakamura Y, Norisuye T. Scattering function for wormlike chains with finite thickness. J Polym Sci, Part B: Polym Phys. 2004;42:1398-407.

34. Pavlov G, Finet S, Tatarenko K, Korneeva E, Ebel C. Conformation of heparin studied with macromolecular hydrodynamic methods and X-ray scattering. Eur Biophys J. 2003;32:437-49.

35. Hayashi K, Tsutsumi K, Norisuye T, Teramoto A. Electrostatic contributions to chain stiffness and excluded-volume effects in sodium hyaluronate solutions. Polym J. 1996;28:922-8.

36. Khan S, Gor J, Mulloy B, Perkins SJ. Semi-rigid solution structures of heparin by constrained X-ray scattering modelling: new insight into heparin-protein complexes. J Mol Biol. 2010;395:504-21.

37. Johansson JA, Halthur T, Herranen M, Soderberg L, Elofsson U, Hilborn J. Build-up of collagen and hyaluronic acid polyelectrolyte multilayers. Biomacromolecules. 2005;6:1353-9.

38. Zhang J, Senger B, Vautier D, Picart C, Schaaf P, Voegel JC, Lavalle P. Natural polyelectrolyte films based on layer-by layer deposition of collagen and hyaluronic acid. Biomaterials. 2005;26:3353-61.

39. Li W, Zhao P, Lin C, Wen X, Katsanevakis E, Gero D, Felix O, Liu Y. Natural polyelectrolyte self-assembled multilayers based on collagen and alginate: stability and cytocompatibility. Biomacromolecules. 2013;14:2647-56. 